\title{
Hodgson's bibliometric report and the reconstruction plan of economics
}

\author{
Yuji Aruka ${ }^{1}$
}

Published online: 6 June 2018

(C) The Author(s) 2018

\begin{abstract}
This article was originally designed to comment an unprecedented bibliometric study of evolutionary economics by Hodgson and Lamberg (Evol Inst Econ Rev 15, 2016). However, Hodgson only reported the historical trajectory and the current situations of evolutionary economics initiated by Nelson and Winter (An evolutionary theory of economic change. Belknap Press of Harvard University Press, Cambridge, 1982). He gave an insightful consideration about the raison d'être of evolutionary economics. According to his opinion, evolutionary economics is still failing to equip the core theories. We naturally agree with his remarks on the current situations around evolutionary economics. We take this opportunity to squarely address the subject about how to insert the core theories into evolutionary economics. We argue that the alternative candidates to be replaced with the main stream core theories are in the following ordering: (1) the theory of production to invalidate myopic optimization, (2) the theory of preference to invalidate myopic optimization, (3) the SMD Theorem to invalidate invisible hand, and (4) the market mechanism to invalidate the efficiency market hypothesis. Needless to say, the alternative theories shown in these arguments imply the reconstruction of economics. Finally, we address the current analysis of bibliometrics.
\end{abstract}

Keywords Reconstruction of economics $\cdot$ Zonotope $\cdot$ Imperfect identification $\cdot$ AI market $\cdot$ Class $4 \cdot$ Bibliometrics

JEL Classification $\mathrm{A} 10 \cdot \mathrm{D} 10 \cdot \mathrm{D} 20 \cdot \mathrm{D} 50 \cdot \mathrm{D} 70$

Yuji Aruka

aruka@tamacc.chuo-u.ac.jp

1 Chuo University, Hachioji, Japan 


\section{Toward a reconstruction plan of economics}

The work of Hodgson and Lamberg (2016) is an unprecedented work on bibliometric analysis of evolutionary economics in its range and scope. First, this analysis retrieved 8474 articles. Second, it separated the development of the time profiles in the subsequent 5 year periods: 1986-1990, 1991-1995, 1996-2000, 2001-2005, and 2006-2010. Thus, this study was able to refer to the present trend and the fashions around the different and cross disciplines. In this sense, the observed facts that Hodgson and Lamberg discovered seem to be almost all reasonable from our institutional overview. Furthermore, in connection with the author's great intelligence, this bibliometric analysis has finally given an insightfully useful perspective to reconstruct economics for us.

In spite of wider penetrations/success over diverse fields except for economics over years, as Hodgson and Lamberg (2016) stated, it must be true that almost no one was successful in replacing the core engine with the main stream's one. His suggestions fortunately coincide with our original plans appeared in Aruka (2017) to revive economics ontologically. This is the reason why I decided to comment on this article. As Hodgson and Lamberg recommended, the raison d'être of evolutionary economics must be the innovation of the core theories of the traditional economics. In other words, the belief of the main stream economics is that everything is at best. On the contrary, our belief is that everything is evolving. We continue to comprehend that "evolutionary economics" as "economics to be evolved". In this point of view, first of all, the reconstruction of political economy mattered. In this sense, Japan Association for Evolutionary Economics (JAFEE) adopted another purpose against the standard definitions of evolutionary economics at the very outset of the foundation. She was sustaining her effort to construct the core theories, such as the theory of value, as an alternative to the main stream. Based on that, Shiozawa $(2007,2015)$ used to sub-tropical geometry to provide a general proof of international trade theory of value in the case of the transaction of three commodities including intermediate goods among three countries. This is the great innovation since Ricardian theory of value. Due to Shiozawa's contributions, the existing theory of comparative cost and its marginal version has lost their justification.

Once upon a time at Cambridge UK, Kaldor (1955) purposed constructing "alternative theories" of distribution to replace the neoclassical marginal productivity theory. He and his followers' attempts have never been successful at rejecting the existing core theory of marginal productivity with their doctrine. The Sraffian theory of value survived longer in being an alternative candidate in value and distribution. However, the classical principles of political economy have been thoroughly replaced with equilibrium economics. Equilibrium economics always depends on the belief that everything is at best, even in serious depressions. It is interesting to note that the Leibzian idea of everything is at best was criticized by Voltaire (2015).

We regard evolution as an idea of the antithesis against the everything-at-itsbest proposition. The real world of evolution is then irrelevant to the grand design of God. Biological evolution does not contain an original intention to optimize 
the whole system to coordinate other organs consistently, at least at the outset of mutation. In this context, evolution is rather motivated by creative coincidence in Mainzer (2007) as a consecutive source of innovation. The same mechanism that causes the skin's blood to condense causes blood condensation in the brain. In general, evolution does not give any normative optimality at all. Thus, the use of the idea of evolution becomes a strong support to the construction of the core theories of production and preference without the idea of optimization and human rationality. ${ }^{1}$

The main stream economics updated herself to survive. Despite of Hirofumi Uzawa's (1928-2014) expectation, the rational expectational hypothesis internationally spread in the economics departments. As demonstrated in Aruka (2015), it is noted that the idea of bounded rationality is considered the raison d'être of rationality. That is to say, bounded rationality is not any modification of rational economics at all but a refined form of it. Reality is always skewed and polluted, because the secular world is not the world of God if we are faithful to the tradition of Thomas Aquinas.

\section{The preliminaries to replace the core theories of economics}

We first mention the recent prospects to innovate the core theories of economics. Hildenbrand $(1981,1994)$ has already given us a set of great hints to innovate the theories in both production and consumer preference. Although my exposition is not exhaustive, in the following, we will refer to how several eminent scholars prepared for the alternative theories. In my opinion, the present time is overdue to innovate on the traditional ideas. Now, we mention the main points and illustrate them very shortly.

1. Theory of production to invalidate myopic optimization.

(a) Hildenbrand (1981) and the zonotope set of production.

(b) A fallacy of the specification on a particular technique and the zonotope set of the international trade.

2. Theory of preference to invalidate myopic optimization.

(a) A sufficient condition to confirm the positive income effect in Hildenbrand (1994).

(b) The utility theory of imperfect identification in Saari $(1959,2005)$.

3. The SMD Theorem to invalidate the invisible hand.

(a) A generalization on SMD theorem in Saari (1992).

(b) Aggregation and choice paradoxes.

\footnotetext{
${ }^{1}$ It is noted that there are two kinds of rationality: human rationality and algorithm rationality.
} 
4. The market mechanism to invalidate the efficiency market hypothesis.

(a) The U-Mart system as an ontological construction of the exchange system by AI.

(b) Class 4 of Fully Random Iterated Automata (FRIA).

\subsection{Theory of production to invalidate myopic optimization}

\subsubsection{Hildenbrand (1981) and zonotope set of production}

There were few to understand the importance Hildenbrand (1981). Dosi et al. (2016) and Scuola Superiore Sant'Anna noticed its importance. Hildenbrandt proved that the short-run production function which was empirically measured did not fulfil the contours of the neoclassical production function, but, rather, the form of zonotopic set of the discrete basic activities of production in a Minkowski space. This set geometrically forms a shape like a rugby ball. Most importantly, the use of zonotopic production set permits us to analyze the recursive property of activities/techniques. Either the neoclassical school or Leontief's input-output analysis was not able to implement a recursive process of production in general.

In short, "[l] eaving the special space restricted by the traditional production function, we can observe various possible combinations to accept institutional effects. Broadening the space, from where technology can be chosen, implies to introduce all possible combinations of activities" (Aruka 2017, p. 410). Dosi et al. (2016) successfully applied this kind of production set to technical progress by calculating the volume of this set. This must be a great progress in the theory of production to invalidate the myopic optimization in production.

\subsubsection{A fallacy of specialization on a particular technique and the zonotopic set of the international trade}

A good example of the benefit of specialization in a particular techniques is the merger or an integration of two basic activities/plants. However, as Aruka (2017) demonstrated, this does not depict the effect of specialization in the international trade between two countries. So far, no one was successful to formulate a general model of international trade of intermediate commodities. This was due to the different rates of distribution among both countries are complicatedly intermingled with the country-wise profile space and the commodity-wise profile space. This interrupted us in confirming equilibrium prices. Fortunately, Shiozawa $(2007,2015)$ gave historically great contributions to find equilibrium in this case. By this proof, it was verified that we never required a particular specification of commodity production for the world efficient production.

This theorem permits us to model the international trade between the same industries, even in equilibrium. Thus, we have also been released also from the neoclassical bindings in the international trade theory. It is also noted that the world production system of three countries and three commodities is exactly the zonotopic set, as shown in Aruka (2017, p. 472) (Fig. 1). 


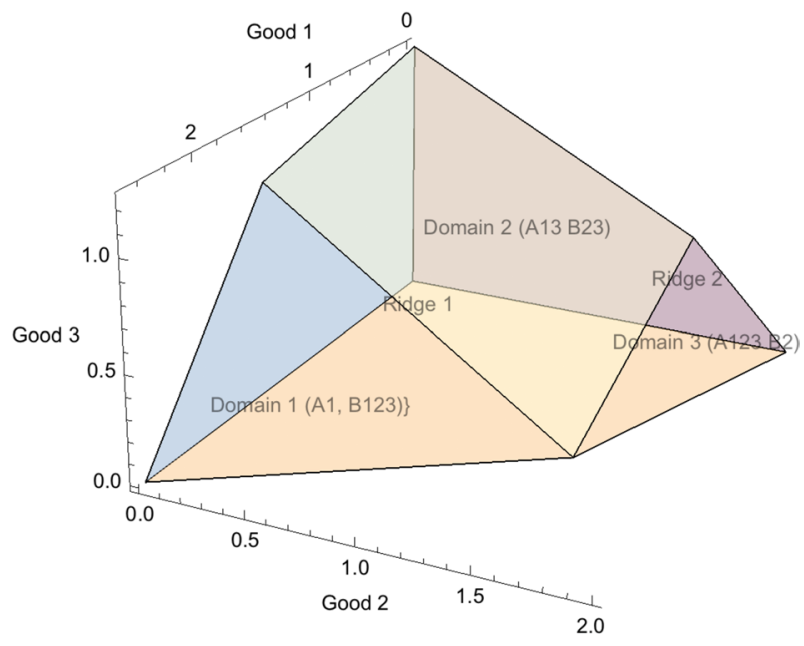

Fig. 1 Shiozawa's diagram of international theory of value. Reproduced from Fig. 4 of Aruka (2017)

\subsection{Theory of preference to invalidate myopic optimization}

\subsubsection{A sufficient condition to confirm the positive income effect in Hildenbrand (1994)}

The great success of Pareto-Slutsky equation (Slutsky 1915) in analyzing consumer demand was given as one of the raison d'être of the main stream economics. However, this analysis never was completed to derive the law of consumer demand, which says that the demand decreases as the price increases. Despite being able to confirm the direction of income effect, surprisingly, many insisted to call Pareto-Slutsky equation the law of demand with a studied nonchalance. This attitude was considerably frivolous. The usual practice to justify Slutsky equation is to impose the assumption of gross substitutability (GS).This assumption is equivalent with respect to the income effect being negligible, despite of the discovery of Giffen effect. It is quite easy to find Giffen effect in the simplest exchange economy of two commodities if the budget is presented in terms of the real endowments of the economy and not using income in terms of monetary units, as shown in Aruka (2015, p. 49). That is to say, the Giffen effect is not an exceptional case. The followers of the General Equilibrium Model were too idle to look for a sufficient condition to establish the direction of sings of the income effect.

Hildenbrand (1994) has smartly given several sufficient conditions to establish the positiveness of income effect. One of these conditions is that as income $x$ increases, the spread of demand $f$, which is the sum of variance and square mean of demand $f$, increase. In other words, the consumption patterns of the richer class become diverse. Here, it is useless to apply a monotheistic idea of myopic optimization. This suggests a great conversion from the neoclassical method of homogeneous agents to the new method of heterogeneous gents. The idea of mathematical 
closeness both in the commodity space and in terms of personal satisfaction must be a fallacy if we envisage the way in which patterns of consumption will be formed in reality. Outside sophisticated mathematical modeling, we need a different idea of consumption such as shown in Aruka (2015, pp. 51-62).

\subsubsection{The utility theory of imperfect identification in Saari (2005)}

The alternative theory of choice must be the probabilistic choice theory of utility given by Saari (1959). However, the generic usage of this theory is almost for a practical one to predict the consumer demand of transportation or sightseeing, including mode choices. This method became well known by McFaden's Nobel Prize in the academic world. When Luce's utility is algebraically considered, this is currently dealt with as the multi-logit model, which can illustrate either successive choices or mode choices. Alternatively, it may be possible to derive the multi-logit model-type utility from the neoclassical assumptions. ${ }^{2}$ A restrictive assumption like independence from irrelevant alternatives is often imposed. However, almost all of such an attempts depend on the assumption that the set/subset of preference is universal.

However, the original version of Saari (1959) does not need to assume universal preference set. In a serial paper of topological geometrical investigation, Saari (2004) showed that imperfect identification of alternative issues could generate paradoxes that are sensitive to a small change of circumstances. Given the preference set/ subsets, it turns out that there are trivially formed probability profiles and ranking profiles. Either in voting or commodity exchanges, the ranking result of candidates depends on a relative position of the preference subset in relation to the probability profile.

It is noted that the ranking results of candidates may be changed, because agent's preferences change. His/her position never changes, but a preference subset to which he/she belongs changes either by forming a coalition of several agents or by the new introduction of an alternative issue, for instance. In short, his/her relative distance from the candidates changes. Thus, such a change of a relative position can be imperfectly identified to give rise any counter-intuitive paradox, thus contradicting myopic optimization.

\subsection{A generalization of the SMD theorem to invalidate invisible hand}

\subsubsection{A generalization on SMD theorem in Saari (1992)}

A serial theorem argued by Sonnenschein (1972, 1973), Mantel (1974), and Debreu 1974) is called the SMD theorem. SMD states that the aggregate excess demand function can always be constructed only by the classical assertions about continuity, homogeneity, and Walras' law. There is no need any other general properties. Hence, the SMD theorem, at first look, seems the most robust theorem to guarantee an equilibrium in any circumstance to justify the invisible hand. Furthermore, many

\footnotetext{
${ }^{2}$ See, for instance, Durlauf $(1997,2000)$.
} 
are convinced that the Walrasian market mechanism is universal almost in any circumstance. Due to this conviction, many are willing to generalize this result in the financial market. This enthusiasm still supports the belief of efficient market hypothesis (EMH) in financial markets.

On the other hand, Saari (1992), in the context of the original Luce theory, carefully inspected this theorem to generalize this theorem and provide an alternative proof to show that to prove a general equilibrium of the market is essentially equivalent to prove the winning process of voting.

[Saari's generalized theorem (Saari 1992)] asserts that almost anything imaginable can happen! Examples can be created where Smith's invisible hand grasps nothing. Using only diagrams of the form used in a freshman economics course, examples can be created that support Adam Smith's story for all pairs of commodities, but, with the same agents, the price dynamic is chaotic for all triplets, then all sets of four commodities have several equilibria where some are attractors and other are not, and then...(Saari 2015, p. 134)

\subsubsection{Aggregation and choice paradoxes}

Roughly speaking, any variation of a purchased commodity changes a next optimal position of the individual. The purchase of "IPad, for instance, can radically change customer demand structure (Saari 2015, p. 134)". Suppose that a subset where this change occurs is separated from a larger subset of commodities. We do not need to know the aggregated excess demand of any larger subset. In short, the aggregated demand function never specifies how a new equilibrium will be generated by what kind of adjustment process. The same reasoning will also be valid to theory of vote, or social choice. The latter theory is often faced with a dead end like voting paradoxes.

In addition, here, the method of aggregation matters. It is quite interesting to see that an intervention of aggregation rather causes to reflect the peculiarities of the particular rule but not the real data (Saari 2015, p. 125).

"Positional elections" (or "positional election rules") are the name attached to election rules where ballots are tallied by assigning points to candidates according to how they are positioned (i.e., ranked) on the ballot. The widely used "plurality vote," where each voter votes for one candidate and the candidate with the largest number of votes wins, assigns one point for a topranked candidate and zero for all others. (Saari 2004, p. 232)

To be sure, we are used to use an extreme evaluation like plurality vote. Therefore, a small positional change of candidates will give rise chaos. 


\subsection{The market mechanism to invalidate the efficiency market hypothesis}

\subsubsection{The U-Mart system as an ontological construction of the exchange system by $A I$}

In the above subsections, we referred to the invalidation of myopic optimization in theoretical point of view. Economics depicted the market as the integration of production and preference. General equilibrium theory is simply one of the pictures to sketch the market economy. Thus, it must be indispensable for us to argue how the market mechanism really is working, without introducing myopic optimizing agents.

The AI market is no longer a virtual market. This is actually a real market. It is not realistic to argue about the stock exchange without referring to the high-frequency transaction (HFT). The computerization of the market does not guarantee a purity of competitiveness of the market. In April 2018, Japan Exchange decided to adopt the policy of the advanced registration of the HFT agent. This may not be a natural outcome for the general equilibrium and/or the invisible hand's supporters.

The AI market based on algorithmic rationality is evidence that the market can accept another principle different from human rationality. Constructing the AI market system is an attempt to construct the market transaction without resorting to the neoclassical principles. In the real market, dominant agents are the so-called technical agents rather than the myopic optimizing and/or rational expectation agent. On the contrary, we can employ the AI market simulator like the U-Mart System ${ }^{3}$ to run our experiments without damaging the functional terms structured by the existing entities of the real market.

\subsubsection{The role of randomness/no intelligence in the market}

In an actual market, participating agents are sending orders either randomly or nonintelligently, even though they depend on their own unambiguous strategies. It is also noted that purely random orders often bring the best performance in the market. Thus, the market system may be a system with many redundancies. ${ }^{4}$

In this century, it turned out that the automaton of the Wolfram rule 110 fulfilled the criteria of Turing completeness. This is among major problems that continued to interest Stephen Wolfram..$^{5}$ Now, the rule 110 and the similar rules are being explored. The cellular automaton has a simple structure, but it is potent in terms of generating complicated behavior like Class 4:

\footnotetext{
3 The U-Mart system is an artificial intelligent futures transaction system of long-running lifetime initiated by Japanese computer scientists since 1998 (See Aruka 2015, pp. 111-112) and Shiozawa et al. (2008).

4 This part depends on Aruka's mimeo paper (Aruka 2018).

5 In particular, see A New Kind of Science (Wolfram 2002).
} 
Class 4 Nearly, all initial patterns evolve into structures that interact in complex and interesting ways, with the formation of local structures that are able to survive for long periods of time. ${ }^{6}$

Now, we accommodate the idea of Class 4 to the market system. In the market, at first, the participants of various types are locally formed, and then, they mutually interact in complex and interesting ways, with the formation of local structures that are able to survive for long periods of time. The Wolfram ICA simulations examined a kind of attractor formation. Conversely, in the market experiment of the U-Mart system, we often observe a certain correspondence generated in a relationship between an initial strategy configuration (ISA) and its final performance configuration (IPA). A final performance is represented by some special form, like attractor. We can examine whether a final performance configuration is wired to its initial strategy configuration or not. A similar set of strategies, in spite of different environments and experimental modes of the market, may fall into a similar result. Irrespective of the rigid assumption of myopic optimization or rational expectation, we can run the analysis of the market.

\section{Some technical comments on the network analysis of cocitations}

As referenced at the beginning, Hodgson and Lamberg (2016) has given a considerably extensive work on a bibliometric analysis of evolutionary economics in a broader range. Needless to say, this work must be the most reliable work to understand the historical transition and the current situation of the profiles of evolutionary economics.

This work observed the interaction of researchers in terms of the cocitation network. The impact factor (IF) is also a well-known index of the cocitation network among journals. This kind of ranking has deeply penetrated the mind of people to reinforce the ambition of people along the current direction of ranking. Now, we must know that there is not so much difference between the secular popularity and the academic popularity. Given the network analysis, we can examine the commonality.

First, it is noted that Hodgson and Lamberg (2016) used Sitkis as a bibliometric software, as in Schildt (2002), and applied it to the Social Science Citation Index (SSCI) of the Thomson-Reuters Web of Science. Currently, we notice that we can easily use a new freeware entitled CiNetExplorer. This freeware is provided by Nees Jan van Eck and Ludo Waltman, Centre for Science and Technology Studies (CWTS) at Leiden University (van Eck and Waltman 2014). We can enjoy and utilize CiNetExplorer with the Web of Science database. In the following, it may then be useful for many readers for us to illustrate very briefly what the cocitation network is.

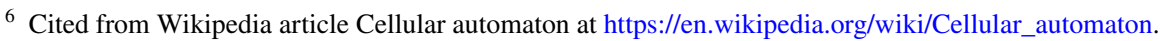




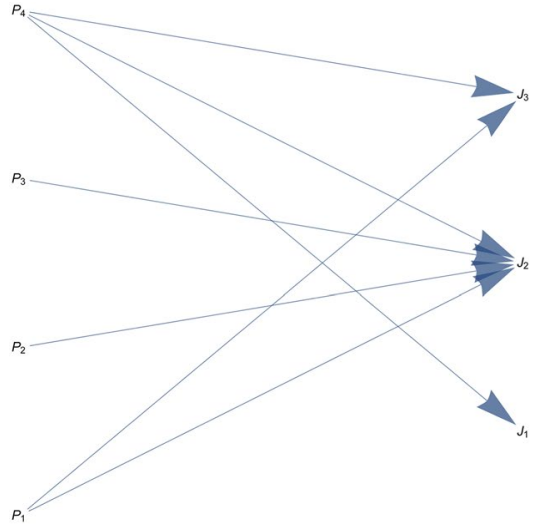

(a) A network of coci-tation

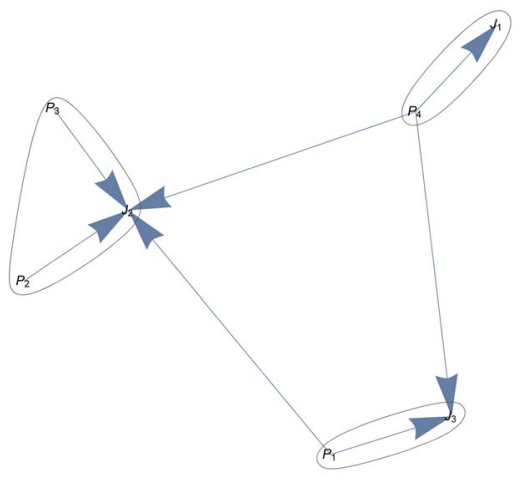

(b) Community plot of the network (a)

Fig. 2 Elementary example 1

To understand the relationship of cocitations, it is often used to use the good hubs and the good authorities relation. Here, we employ the adjacency matrix $a$. By accepting the case of bilateral cocitation for any pair, the cocitation measure will be classified either to identify common ancestors or common descendants. The cocitation matrix to measure common ancestors is defined as follows: ${ }^{7}$

$$
a^{T} a-\text { Diagonal } a^{T} a_{i i}
$$

Figure 2 has shown the so-called relationship of hubs $P_{1}, P_{2}, P_{3}, P_{4}$ and authorities $J_{1}, J_{2}, J_{3}$. The mathematical expression of this relationship will be represented by the cocitation matrix $(\mathrm{CM})$ :

$$
C M=\left(\begin{array}{lll}
0,0,0,0,0,0,0 & -P_{1} \\
0,0,2,0,0,0,1 & -J_{2} \\
0,2,0,0,0,0,1 & -J_{3} \\
0,0,0,0,0,0,0 & -P_{2} \\
0,0,0,0,0,0,0 & -P_{3} \\
0,0,0,0,0,0,0 & -P_{4} \\
0,1,1,0,0,0,0 & -J_{1}
\end{array}\right.
$$

This matrix then indicates the cocitation between the vertices $J_{2}$ and $J_{3}$ that share their common ancestors $P_{1}$ and $P_{4}$. In terms of the cocitation matrix, we can verify

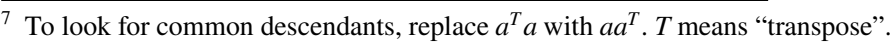




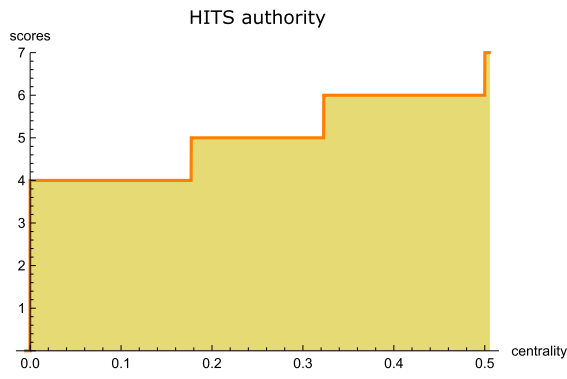

(a)

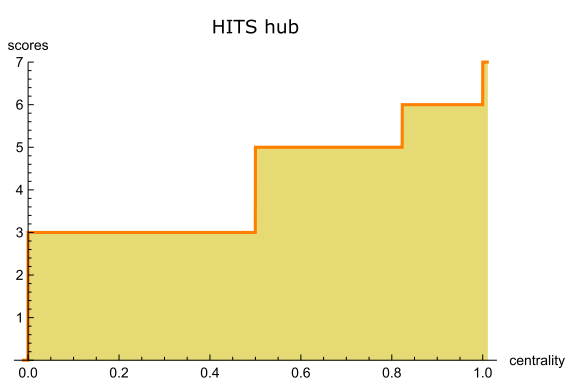

(b)

Fig. 3 Score distributions in elementary example 1

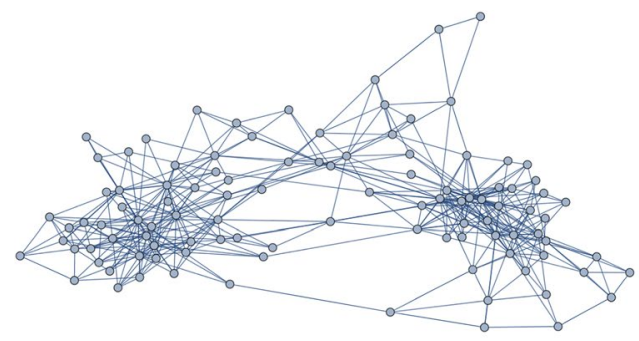

(a) A network of coci-tation

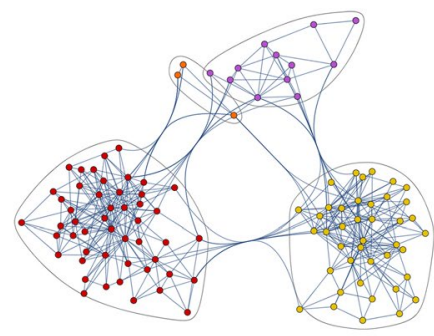

(b) Community plot of the network (a)

Fig. 4 US politics books

them using the second row and the third column (or the third row and the second column).

Now, we have prepared for hit algorithm to estimate hits scores which is the importance of the existing nodes/vertices. In our example, the hit centralities are calculated as follows:

$$
\begin{aligned}
\text { Authority centrality } & =(0,0.5,0.322876,0,0,0,0.177124) \\
\text { Hub centrality } & =(0.822876,0,0,0.5,0.5,1,0)
\end{aligned}
$$

By referring to Wolfram featured example, ${ }^{8}$ we reproduce the scores distribution each centrality (Fig. 3).

The first example certainly revealed a fundamental structure of the cocitation network. However, there are too few vertices to grasp a macroscopic property of the hit centralities. We may examine another example with a relatively bigger size that is

\footnotetext{
${ }^{8}$ Compare Centrality in Citation Networks http://reference.wolfram.com/language/example/Compa reCentralityInCitationNetworks.html?view=all .
} 


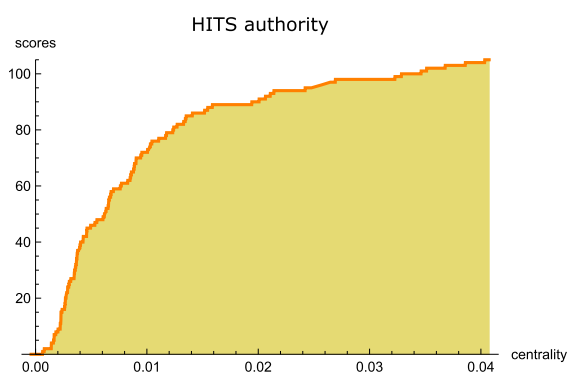

(a)

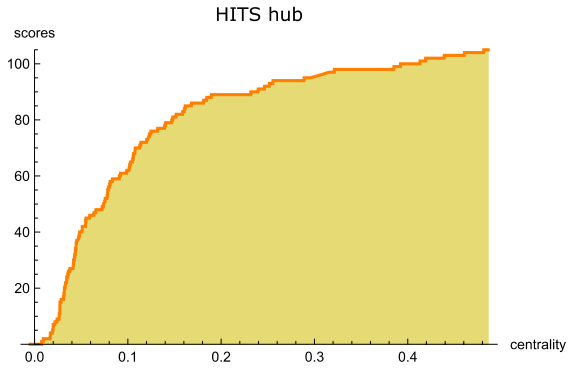

(b)

Fig. 5 US politics books

Table 1 EIER annual citations analyzed by Web of Science in 2018

\begin{tabular}{ll}
\hline Pub year & Records \\
\hline 2015 & 27 \\
2016 & 27 \\
2017 & 30 \\
\hline
\end{tabular}

The total number of citations to all results found in the results set minus any citation from articles in the set (sum of times cited without self-citations field)

cited from Mathematica's sample data entitled ExampleData: "USPoliticsBooks" in "NetworkGraph". 9 These data provide 105 books on politics in the US. By applying the same procedure stated above to these data, we produce the network graph, its community plot, and the scores distribution each centrality of this US politics data.

In the first example, it is seen that the scores of each centrality increase as each centrality is greater (Fig. 4). In comparison with Figs. 3 and 5, furthermore, we will see that a concentration of score is reinforced as each centrality is greater. In other words, greater centrality will earn a higher score, while a higher score will not imply a greater centrality. Even if a smaller centrality earns a high score, its effect to the network will naturally be smaller. It is noted that these effects may not depend on the kinds of data to be adopted.

Finally, we close this article by referring to the present statistics of Evolutionary and Institutional Economics Review (EIER) cited from the database of Web of Science. In 2004, the JAFEE launched her society journal and then published the first issue of EIER exclusively specialized for an international journal. However, until 2014, we managed to publish it without an international publisher. Yagi (2018) has given an exposition on the history of JAFEE and EIER. This article will help readers to promote the understanding of JAFEE and EIER. In 2015, Springer has been contracted the publication of EIER. Thanks to Springer Nature's contract, EIER statistics has recently come out in the Web of Science, though EIER has not yet fulfilled

\footnotetext{
9 Downloadable from http://reference.wolfram.com/language/ref/ExampleData.html.
} 
the requirements to join in the IF ranking. However, the last 3 years' figures of citations of EIER of Springer version for 2015-2017 are as follows:

The calculation of IF is generally defined as follows:

$$
\mathrm{IF}_{t}=\frac{\text { citations }_{t-1}+\text { citations }_{t-2}}{\text { publications }_{t-1}+\text { publications }_{t-2}} .
$$

Here, $x_{t}$ represents the figure of $x$ at time $t$. Thus, the annual figure of citation stated in Table 1 is eligible to be considered for the ranking competition soon. EIER will be expected to appear in the list of IF in Web of Science.

Open Access This article is distributed under the terms of the Creative Commons Attribution 4.0 International License (http://creativecommons.org/licenses/by/4.0/), which permits unrestricted use, distribution, and reproduction in any medium, provided you give appropriate credit to the original author(s) and the source, provide a link to the Creative Commons license, and indicate if changes were made.

\section{References}

Aruka Y (2018) An examination of market mechanism with redundancies motivated by Turing's rule selection. In: WEHIA2018 to be held at ICU. mimeo, Tokyo

Aruka Y (2015) Evolutionary foundations of economic science: how can scientists study evolving economic doctrines from the last centuries? Springer series: evolutionary economics and social complexity science, vol 1. Springer, Tokyo

Aruka Y (2015) The rise of pure economics under a new form of scholasticism in view of the present socio-economic system. Evol Inst Econ Rev 13(1):3-29

Aruka Y (2017) Some new perspectives on the inter-country analysis of the world production system. Evol Inst Econ Rev 14(2):467-498

Aruka Y (2017) Special feature: preliminaries towards ontological reconstruction of economics -theories and simulations. Evol Inst Econ Rev 14(2):409-414

Debreu G (1974) Excess demand functions. J Math Econ 1(1):15-21

Dosi G, Grazzi M, Marrengo L, Settepanella S (2016) Production theory: accounting for firm heterogeneity and technical change. J Ind Econ 64(4):875-907

Durlauf SN (1997) Statistical mechanics approaches to socioeconomic behavior. In: Arthur WB, Durlauf SN, Lane DA (eds) The economy as an evolving complex system II. Addison-Welsely, Reading, Mass, pp 81-104

Durlauf SN (2000) A framework for the study of individual behavior and social interaction. SSRI Working Paper Series

Hildenbrand W (1981) Short-run production functions based on microdata. Econometrica 49(5):1095-1125

Hildenbrand W (1994) Market demand. Princeton UP, Princeton NJ

Hodgson GM, Lamberg JA (2016) The past and future of evolutionary economics: some reflections based on new bibliometric evidence. Evol Inst Econ Rev. https://doi.org/10.1007/s40844-016-0044-3

Kaldor N (1955-1956) Alternative theories of distribution. Rev Econ Stud 23(2):83-100

Mantel R (1974) On the characterization of aggregate excess demand. J Econ Theory 7(3):348-353

Mainzer K (2007) Kapitel 7: Zufall in Kultur, Wirtschaft, und Gesselschaft. In: Der kreative Zufall: Wie das Neue in die Welt kommt? CH Beck

Nelson RR, Winter SG (1982) An evolutionary theory of economic change. Belknap Press of Harvard University Press, Cambridge

Saari DG (1959) Individual chaotic behavior. Wiley, New York

Saari DG (1992) The aggregate excess demand function and other aggregation procedures. Econ Theory 2:359-388 
Saari DG (2004) Geometry of chaotic and stable discussions. Am Math Mon 111(5):222-230

Saari DG (2005) The profile structure for Luce's choice axiom. J Math Psychol 49:226-253

Saari DG (2015) Social science puzzles: a systems analysis challenge. Am Math Mon 12(1):123-139

Schildt HA (2002) SITKIS: software for bibliometric data management and analysis. Helsinki University of Technology, Espoo

Shiozawa Y (2007) A new construction of Ricardian trade theory a many-country, many-commodity case with intermediate goods and choice of production techniques. Evol Inst Econ Rev 3(2):141-187

Shiozawa Y (2015) International trade theory and exotic algebras. Evol Inst Econ Rev 12(1):177-212

Shiozawa Y, Nakajima Y, Matsui H, Koyama Y, Taniguchi K, Hashimoto F (2008) Artificial market experiments with the U-mart system. Springer series on agent based social systems, vol 4. Springer, Tokyo

Slutsky EE (1915) Sulla teoria del bilancio del consumatore. Giornale degli Economisti 51:1-26

Sonnenschein H (1972) Market excess demand functions. Econometrica 40(3):649-663

Sonnenschein H (1973) Do Walras' identity and continuity characterize the class of community excessdemand functions? Econometrica 6(4):345-354

van Eck NJ, Waltman L (2014) CiNetExplorer:analysing citation patterns in scientific literature. Centre for Science and Technology Studies (CWTS), Leiden University. http://www.citnetexplorer.nl

Voltaire (2015) Candide, ou l'Optimisme (1759) by Voltaire, translated by Tobias Smollett (updated)

Wolfram S (2002) A new kind of science. Wolfram Media, Champaign

Yagi K (2018) Note: Road to evolutionary and institutional economics in Japan—a personal memoire of a decade around its foundation. Evol Inst Econ Rev 15(1) 